\title{
Series médicas en televisión vistas por estudiantes de medicina
}

Emilio Pintor-Holguín, Margarita Rubio-Alonso, Benjamín Herreros Ruiz-Valdepeñas, Octavio J. Corral-Pazos de Provens, Luis Buzón-Rueda, Enrique Vivas-Rojo

Introducción. Después del éxito de la serie televisiva Urgencias, ha proliferado en estos últimos 10 años la producción y emisión de 'series de médicos'. Pretendemos analizar si nuestros estudiantes de medicina ven estas series, por qué les atraen y si les influyen de algún modo.

Sujetos y métodos. Se incluyeron todos los alumnos de primer, segundo y tercer cursos del Grado de Medicina en la Universidad Europea de Madrid durante el año académico 2010-2011. Se realizó una encuesta sobre la visualización y percepción de las series de médicos de mayor audiencia en España: Urgencias, Hospital Central, House y Anatomía de Grey. El trabajo de campo se hizo en marzo de 2011.

Resultados. Cumplimentaron la encuesta 213 alumnos (53\% de la muestra), con una edad media de 19,5 años. El 70,8 \% eran mujeres. El $84 \%$ veían con frecuencia de una a tres de las series planteadas, siendo la más seguida House (68,1\%). Lo que atrae al $70 \%$ es tanto el contenido médico como la trama argumental no médica. La credibilidad médica que dan a las series es alta, por encima de 2,5 (en una escala de 1 a 5), siendo Urgencias la más creíble para ellos. Para un 26,8\%, el hecho de ver estas series habría podido influir en su elección profesional.

Conclusiones. Dado que nuestros alumnos de medicina ven con frecuencia este tipo de series y pueden influirles en algún sentido, nuestro deber como docentes es estimular una visión crítica del contenido médico-científico que en ellas aparece.

Palabras clave. Estudiantes de medicina. Series de médicos. Series de televisión.

\section{Medical television series seen by students of medicine}

Introduction. After the success of television series ER (Emergency Room), over the past 10 years the production and issuance of 'medical series' has proliferated. We intend to analyze if our medical students see this series, why and if they are influenced in some way by seen them.

Subjects and methods. We included all students in 1st, 2nd and 3rd Degree in Medicine at the Universidad Europea de Madrid for the 2010-2011 academic year. The survey included a series of questions about viewing and perceptions of the series of doctors with the largest audience in Spain: ER, Hospital Central, House MD and Grey's Anatomy. Fieldwork was conducted in March 2011.

Results. 213 students completed the survey (53\% of the sample) with a mean age of 19.5 years. $70.8 \%$ were women. $84 \%$ of students reported having seen frequently from one to three of the series raised, the most followed was House MD (68.1\%). $70 \%$ of them see this series because medical and non-medical plot. The medical credibility that they give this series is high, above 2.5 (on a scale of 1 to 5), being the most credible ER. $26.8 \%$ claimed that the display of these series had been able to influence their career choice.

Conclusions. Because our medical students often see this type of series and can influence them in some sense, our duty as teachers is to encourage a critical view of medical and scientific content that appears on them.

Key words. Medical students. TV medical dramas. TV series.

\section{Introducción}

Aunque en la década de los cincuenta comenzaron a realizarse y emitirse en Estados Unidos las primeras series de televisión con contenido médico (City Hospital o The Doctor), no sería hasta los años seten- ta cuando algunas de ellas (Centro Médico y Marcus Welby, doctor en medicina) llegaron a las pantallas de nuestro país. Desde entonces hasta la actualidad se han emitido en todo el mundo más de 70 series de televisión con temática médica donde se ha producido una evolución del comportamiento y carac-
Departamento de Especialidades Médicas Aplicadas. Facultad de Ciencias Biomédicas de la Salud. Universidad Europea de Madrid. Madrid, España.

Correspondencia: Dr. Emilio Pintor Holguín. Departamento de Especialidades Médicas Aplicadas. Facultad de Ciencias Biomédicas de la Salud. Universidad Europea de Madrid. Tajo, s/n. E-28670 Villaviciosa de Odón (Madrid).

E-mail:

emilio.pintor@uem.es

Conflicto de intereses: No declarado.

Conflict of interests: None declared.

(C) 2012 Educación Médica 
Tabla I. Cuestionario sobre series de televisión relacionadas con la salud.

Edad:

Sexo (Varón / Mujer)

Estudios que está cursando (si está entre dos cursos, señale el más elevado)

Medicina

$1^{\text {er. }}$ curso

2.․ㅜ curso

$3^{\text {er. }}$ curso

De las siguientes series que se han emitido en canales españoles en los últimos 3-4 años, señale de 1 a 5 la frecuencia con que las ha visto (1: nunca; 2 : pocas veces; 3 : frecuentemente; 4: casi siempre; 5: siempre):

$\begin{array}{llllll}\text { Urgencias } & 1 & 2 & 3 & 4 & 5 \\ \text { Hospital Central } & & & & & \\ \text { House } & & & & & \\ \text { Anatomía de Grey } & & & & \end{array}$

La justificación de que le gusten o vea estas series es su contenido medico, no médico 0 ambos. Marque sólo en las que ha señalado 3, 4 o 5 en la pregunta anterior:

$\begin{array}{lll} & \text { Contenido médico } & \text { Drama-comedia } \\ \text { Hospital Central } & & \\ \text { House } & & \\ \text { Anatomía de Grey } & & \end{array}$

Según su criterio, puntúe de 1 a 5 las siguientes series que ha visto (en que haya marcado 3, 405 en la primera pregunta, según su veracidad científico-médica, la que se ajuste más a la realidad) (1: poco; 5: mucho):

$\begin{array}{llllll}\text { Urgencias } & 1 & 2 & 3 & 4 & 5 \\ \text { Hospital Central } & & & & & \\ \text { House } & & & & \\ \text { Anatomía de Grey } & & & \end{array}$

¿Cree qué la visualización de este tipo de series ha podido influir en su elección profesional dentro del campo sanitario? (Sí / No)
Pero, ¿qué imagen ofrecen estas series sobre los médicos y la medicina de nuestro tiempo? ¿Se ajustan a la realidad diaria de la práctica clínica o son pura ficción? La importancia de este tipo de series se ha analizado desde el punto de vista periodístico y se han realizado tesis doctorales sobre el tema [5].

La opinión de los médicos sobre ellas no se ha hecho esperar, aunque hay cierta controversia sobre los mensajes que se ofrecen en esas series. La mayor parte de los estudios publicados se han dirigido a valorar cómo se presenta en la ficción la resucitación cardiopulmonar. Mientras que en 1996, poco después de que se estrenaran las series televisivas ER y Chicago Hope, la prestigiosa revista The New England Journal of Medicine publicaba un artículo alertando sobre el irreal alto porcentaje de éxito de las resucitaciones cardiopulmonares que aparecían en estas series [6], después se ha publicado que en la series médicas británicas [7] (Casualty, Cardiac arrest, Medics) o belgas [8] (Spoed), la supervivencia inicial es más cercana a la realidad, aunque no así los grupos de pacientes ni las causas de parada cardiorrespiratoria que se muestran. Un artículo más reciente, que analiza Casualty, Holby City, Anatomía de Grey y Urgencias, va en el mismo sentido [9].

En nuestro país, el éxito de audiencia y difusión en la sociedad de este tipo de programas ha hecho incluso que la Organización Médica Colegial haya tomado cartas en este asunto. Así, la Comisión Central de Deontología de dicha institución emitió un informe [10] en que alertaba de los 'riesgos' de desinformación o mala información que puede recibir la población general al visualizar este tipo de series. Entre ellas podríamos señalar: crear falsas expectativas en los pacientes, ser poco realistas (deberían aparecer más ancianos y menos niños), realizar menos exploraciones sofisticadas, menos recuperaciones milagrosas, menos tratamientos experimentales...

Los objetivos del presente estudio han sido analizar en los estudiantes de medicina algunos aspectos de las series médicas de televisión emitidas en nuestro país:

- Analizar cuántos de ellos ven con asiduidad o frecuencia las series médicas que se emiten.

- Saber cuáles de las estas series planteadas son la más vistas y por qué las ven.

- Valorar el grado de credibilidad o rigor científico que les dan a las series que ven con frecuencia.

- Conocer el porcentaje de alumnos en los que la visión de este tipo de series ha podido influir de algún modo en su elección profesional. 


\section{Sujetos y métodos}

Se realizó un estudio observacional y transversal en el que se incluyeron a todos los alumnos que realizaban estudios de Grado de Medicina en el curso académico 2010-2011 en la Facultad de Ciencias Biomédicas de la Universidad Europea de Madrid. En ese curso había matriculados un total de 400 alumnos de medicina: 240 alumnos en primer curso, 105 en el segundo y 55 en el tercero.

Los días seleccionados para cumplimentar los cuestionarios se eligieron al azar durante el mes de marzo de 2011. Los cuestionarios fueron cumplimentados por los alumnos que asistieron ese día a clase y que voluntariamente decidieron hacerlo. Se les informó previamente de los objetivos del estudio y del carácter voluntario y anónimo del cuestionario.

Se utilizó un cuestionario (Tabla I) de elaboración propia en el que se incluyeron variables generales (edad, sexo y curso que estudiaban) y una serie de preguntas relacionadas con las series televisivas sobre médicos que se han emitido en el último lustro en España. Se eligieron cuatro series de televisión: Urgencias (emitida por TVE desde 1999), House y Anatomía de Grey (emitidas ambas por Cuatro desde el año 2006) y Hospital Central (emitida por Telecinco desde el año 2000), por ser las que más audiencia televisiva han tenido en España [4].

Las preguntas de la encuesta se elaboraron de la forma más sencilla posible para facilitar su comprensión. Las preguntas acerca de la frecuencia con que veían las series de televisión mencionadas y la credibilidad que otorgaban al contenido de éstas se midieron mediante una escala Likert de 1 a 5. En el documento de la encuesta se indicó a los alumnos que contestaran a la pregunta sobre la credibilidad sólo si habían visto la serie indicada 'frecuentemente', 'casi siempre' o 'siempre', es decir, si habían contestado 3, 4 o 5 en la escala Likert de la pregunta sobre frecuencia (Tabla I). Se incluyó también una pregunta sobre la justificación o el motivo por el que veían estas series, con tres posibles respuestas ('contenido médico', 'drama/comedia' o 'ambas'), y otra pregunta sobre si el visionado de las series había influido en su decisión de estudiar medicina.

Los datos obtenidos se analizaron utilizando el programa estadístico SPSS. Se realizó un análisis estadístico descriptivo y un análisis comparativo de los resultados por curso. Para la descripción de frecuencias, las variables cualitativas se expresaron como porcentaje y las variables cuantitativas (edad)
Figura 1. Frecuencia con la que se ven cada una de las series (1: nunca; 5: siempre).

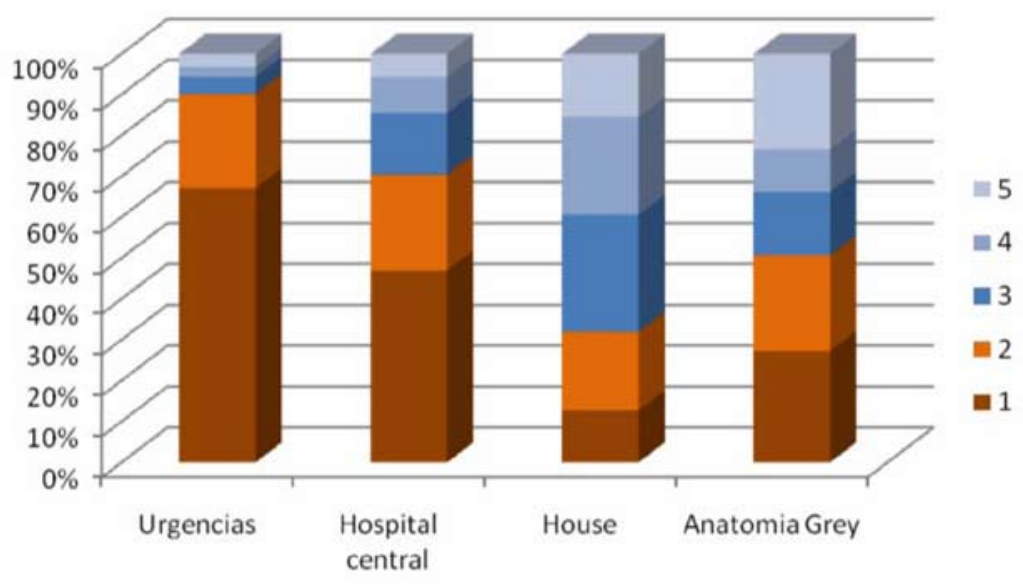

Figura 2. Credibilidad científica que dan los alumnos a cada una de las series (1: nada; 5: mucho)

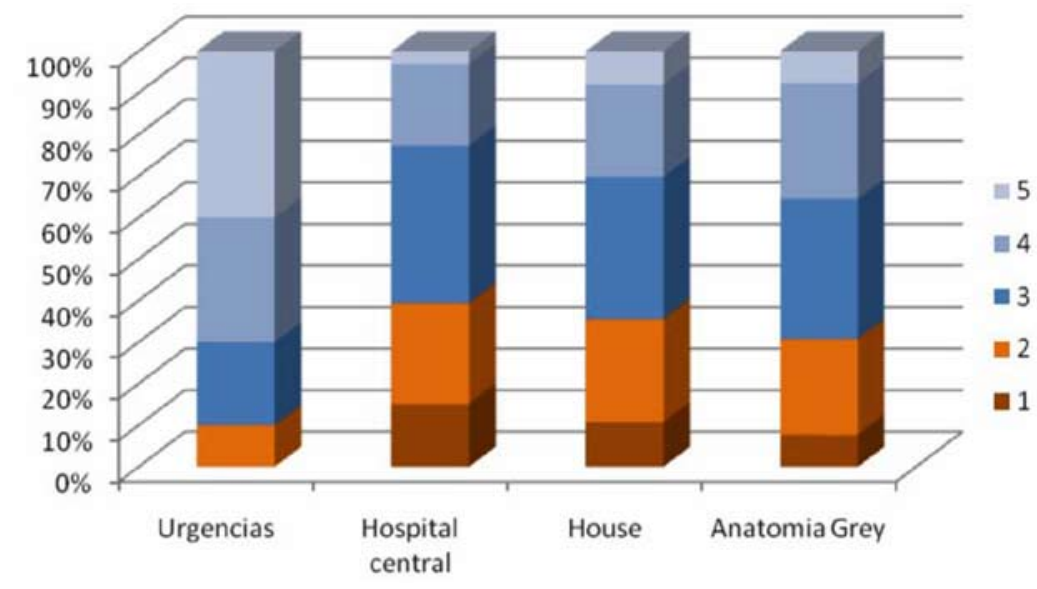

se expresaron como media \pm desviación estándar. Para las variables medidas mediante una escala Likert de 1 a 5 se utilizó la mediana como medida de tendencia central. Para estas variables también se calculó el porcentaje de alumnos que contestó a cada una de las opciones. Para la comparación de los porcentajes de respuesta entre los distintos cursos se utilizó el test de chi al cuadrado. Se consideraron estadísticamente significativos valores de $p<0,05$. 
Tabla II. Frecuencia de visión de las series en función del curso.

\begin{tabular}{|c|c|c|c|c|c|}
\hline & & $\begin{array}{l}1^{\text {er. curso }} \\
(n=128)\end{array}$ & $\begin{array}{l}2 . .9 \text { curso } \\
(n=63)\end{array}$ & $\begin{array}{l}3^{\text {er. curso }} \\
(n=22)\end{array}$ & $p$ \\
\hline \multirow{5}{*}{ Urgencias } & 1 & $94(73,4 \%)$ & $37(58,7 \%$ & $12(54,5 \%)$ & \multirow{5}{*}{0,24} \\
\hline & 2 & $24(18,8 \%)$ & $18(28,6 \%)$ & $7(31,8 \%)$ & \\
\hline & 3 & $6(4,7 \%)$ & $3(33,3 \%)$ & 0 & \\
\hline & 4 & $2(1,6 \%)$ & $2(3,2 \%)$ & $1(4,5 \%)$ & \\
\hline & 5 & $2(1,6 \%)$ & $3(4,8 \%)$ & $2(9,1 \%)$ & \\
\hline \multirow{5}{*}{$\begin{array}{l}\text { Hospital } \\
\text { Central }\end{array}$} & 1 & $68(53,1 \%)$ & $25(39,7 \%)$ & $7(31,8 \%)$ & \multirow{5}{*}{0,28} \\
\hline & 2 & $26(20,3 \%)$ & $17(27 \%)$ & $7(31,8 \%)$ & \\
\hline & 3 & $17(13,3 \%)$ & $9(14,3 \%)$ & $6(27,3 \%)$ & \\
\hline & 4 & $12(9,4 \%)$ & $6(9,5 \%)$ & $1(4,5 \%)$ & \\
\hline & 5 & $5(3,9 \%)$ & $6(9,5 \%)$ & $1(4,5 \%)$ & \\
\hline \multirow{5}{*}{ House } & 1 & $19(14,8 \%)$ & $6(9,5 \%)$ & $2(9,1 \%)$ & \multirow{5}{*}{0,66} \\
\hline & 2 & $23(18,0 \%)$ & $12(19,0 \%)$ & $6(27,3 \%)$ & \\
\hline & 3 & $34(26,6 \%)$ & $21(33,3 \%)$ & $6(27,3 \%)$ & \\
\hline & 4 & $35(27,3 \%)$ & $13(20,6 \%)$ & $3(13,6 \% 9$ & \\
\hline & 5 & $17(13,3 \%)$ & 11 (17,5\%) & $5(22,7 \%)$ & \\
\hline \multirow{5}{*}{$\begin{array}{l}\text { Anatomía } \\
\text { de Grey }\end{array}$} & 1 & $39(30,5 \%)$ & $11(17,5 \%)$ & $8(36,4 \%)$ & \multirow{5}{*}{0,62} \\
\hline & 2 & $26(20,3 \%)$ & $18(28,6 \%)$ & $6(27,3 \%)$ & \\
\hline & 3 & $21(16,4 \%)$ & $10(15,9 \%)$ & $2(9,1 \%)$ & \\
\hline & 4 & $12(9,4 \%)$ & $8(12,7 \%)$ & $2(9,1 \%)$ & \\
\hline & 5 & $30(23,4 \%)$ & $16(25,4 \%)$ & $4(18,2 \%)$ & \\
\hline \multicolumn{2}{|c|}{$\begin{array}{l}\text { Influencia en la decisión } \\
\text { de estudiar medicina }\end{array}$} & $29(22,7 \%)$ & $24(38,1 \%)$ & $4(18,2 \%)$ & 0,048 \\
\hline
\end{tabular}

\section{Resultados}

\section{Descripción de la muestra}

Los alumnos que realizaron la encuesta y, por tanto, se incluyeron en el estudio fueron 213 (el 53\% de los 400 que estudiaban medicina ese año): 128 de los 240 (53\%) de primer curso, 63 de los 105 (60\%) de segundo curso y 22 de los 55 (40\%) de tercero. La mayor parte de ellos fueron mujeres $(n=151$; $70,8 \%)$ y el resto, hombres $(n=62 ; 29,2 \%)$. La edad media del grupo fue de 19,5 $\pm 1,7$ años (rango: 1829 años).

\section{Resultados de la encuesta}

Las respuestas de los alumnos a las preguntas sobre frecuencia de visionado de las series (escala de 1 a 5) mostraron que de las cuatro series planteadas, la que con mayor frecuencia veían los estudiantes de medicina, considerando como tal si puntuaron de 3 a 5, fue House, con un 68,1\%, seguida de Anatomía de Grey, con un 49,3\% (Fig. 1). En esta pregunta sobre frecuencia, en la serie House la mediana fue de 3, para Anatomía de Grey fue de 2, para Hospital Central fue de 2 y para Urgencias fue de 1 . En cuanto al número de series vistas con frecuencia $(3,4$ o 5 en la escala), un $17 \%$ de los alumnos veían tres de las cuatro series planteadas, un 36\% veían dos series y un $31 \%$ veían sólo una serie. Del total de alumnos encuestados, un 15\%, afirmaban no seguir ninguna de las series.

Respecto a la justificación que los alumnos esgrimieron para ver estas series, los resultados fueron: para la serie Urgencias, contestaron el 'contenido médico' un 15\%, el 'contenido drama/comedia' (situaciones laborales, sociales, de pareja...) un 15\%, y 'ambas' un 70\%. Para la serie Hospital Central, contestaron un $10,8 \%, 27,7 \%$ y $61,5 \%$, respectivamente. Para la serie House, un $17 \%, 13,6 \%$ y $69,4 \%$, respectivamente, y para Anatomía de Grey, un 2,8\%, $29 \%$ y $68,2 \%$, respectivamente.

En cuanto a la credibilidad científica (si la ficción médica se acerca o no a la realidad clínica) que otorgan los alumnos a estas series, en la figura 2 se muestran las respuestas para cada una de las series. La más creíble para ellos fue Urgencias, de modo que el $90 \%$ de quienes la veían con frecuencia contestaron 3, 4 o 5 en la pregunta sobre credibilidad. Sobre la credibilidad de Anatomía de Grey, el $69,3 \%$ contestaron 3, 4 o 5 en esta pregunta; acerca de House, el 64,4\%, y sobre Hospital Central, el 60,6\%. Para la serie Urgencias, la mediana en la pregunta sobre credibilidad fue de 4, y para el resto de las series, de 3. En las tablas II, III y IV se muestran los resultados de la encuesta por cursos. Sólo encontramos diferencias estadísticamente significativas en las respuestas respecto a la credibilidad de la serie House: contestaron 4 o 5 a esta pregunta el 71,4\% de los alumnos de tercero, el 32,6\% de los alumnos de segundo y el $21,5 \%$ de los alumnos de primero.

Por último, a todos los alumnos se les hizo la pregunta sobre si la visualización de esas series podía haber influido en algún sentido en la elección de su carrera profesional: 57 alumnos $(26,8 \%)$ respondieron afirmativamente.

Se observaron diferencias estadísticamente significativas $(p=0,048)$ en la pregunta sobre la influencia que las series habían ejercido a la hora de elegir los estudios de medicina (Tabla II). El 38,1\% de los estudiantes de segundo curso, el $22,7 \%$ de los de primero y el $18,2 \%$ de los de tercero contestaron que habían influido. 


\section{Discusión}

En este estudio, en el que por primera vez se analizan las series televisivas 'de médicos' desde la perspectiva de los estudiantes de medicina de los tres primeros cursos, se pone de manifiesto que este tipo de series atraen a nuestros estudiantes: el $84 \%$ ven 'casi siempre' o 'siempre' de una a tres series de las cuatro que se plantearon, y además consideran que la mayor parte de ellas tienen una 'calidad científico-médica' aceptable.

A nuestros estudiantes de medicina les gusta ver este tipo de series y coinciden con los gustos televisivos de cualquier otro adolescente de nuestro país. Ven sobre todo House, Anatomía de Grey y, en menor medida, Hospital Central, datos que coinciden con los índices de audiencia globales [11]. La menor visualización de Urgencias tiene su justificación en que es una serie que lleva muchos años en pantalla y ya no se emite en un horario de máxima audiencia en la primera cadena de TVE [3].

Las dos series más vistas por nuestros alumnos son House y Anatomía de Grey. Se han hecho estudios sobre el impacto mediático y social que pueden ejercer estas series y se ha criticado no tanto su contenido médico, sino el estereotipo del médico que se ofrece en ellas $[12,13]$.

La credibilidad médica de estas series es un dato controvertido en la actualidad. La mayor parte de los estudios realizados se relacionan con la resucitación cardiopulmonar y en series que no se han emitido en nuestro país [6-9]. En España se ha posicionado la Organización Médica Colegial alertando de la posible información errónea que se trasmite a la sociedad [10], aunque otras instituciones, como la Sociedad Española de Neurología, elogian la posible utilidad en favorecer la educación sanitaria de la población general [2].

Otro dato interesante de nuestro estudio es que el $26,8 \%$ de nuestros estudiantes dicen haber sido influidos en algún sentido a elegir medicina por la visualización de estas series [14]. Soria et al [15] realizaron uno de los pocos trabajos hechos en España que, sin versar directamente sobre el tema, aportan información al respecto; aunque los autores concluían que 'los estudiantes de medicina tienen un alto grado de vocación social', reconocían la existencia de 'condicionantes externos' a la hora de elegir la carrera. En concreto, el $11 \%$ de los encuestados admitieron la influencia de la televisión. Un artículo de Ballenato [16] subrayaba las 'influencias decisivas' del contexto sociocultural, entre las cuales se encontrarían las series de televisión, a la hora de escoger carrera, y concluía que 'las actividades
Tabla III. Credibilidad de las series en función del curso.

\begin{tabular}{|c|c|c|c|c|c|}
\hline & & $\begin{array}{l}1^{\text {er. }} \text { curso } \\
(n=128)\end{array}$ & $\begin{array}{c}2.9 \text { curso } \\
(n=63)\end{array}$ & $\begin{array}{c}3^{\text {er. }} \text { curso } \\
(n=22)\end{array}$ & $p$ \\
\hline \multirow{5}{*}{ Urgencias } & 1 & 0 & 0 & 0 & \multirow{5}{*}{0,08} \\
\hline & 2 & 0 & $1(12,5 \%)$ & $1(33,3 \%)$ & \\
\hline & 3 & $3(33,3 \%)$ & $1(12,5 \%)$ & 0 & \\
\hline & 4 & $5(55,6 \%)$ & $1(12,5 \%)$ & 0 & \\
\hline & 5 & $1(11,1 \%)$ & $5(62,5 \%)$ & $2(66,7 \%)$ & \\
\hline \multirow{5}{*}{$\begin{array}{l}\text { Hospital } \\
\text { Central }\end{array}$} & 1 & $7(18,9 \%)$ & $2(9,5 \%)$ & $1(12,5 \%)$ & \multirow{5}{*}{0,60} \\
\hline & 2 & $10(27,0 \%)$ & $3(14,3 \%)$ & $3(37,5 \%)$ & \\
\hline & 3 & $12(32,4 \%)$ & $9(42,9 \%)$ & $4(50,0 \%)$ & \\
\hline & 4 & $7(18,9 \%)$ & $6(28,6 \%)$ & 0 & \\
\hline & 5 & $1(2,7 \%)$ & $1(4,8 \%)$ & 0 & \\
\hline \multirow{5}{*}{ House } & 1 & $11(12,4 \%)$ & $3(6,5 \%)$ & $2(14,3 \%)$ & \multirow{5}{*}{0,01} \\
\hline & 2 & $26(29,2 \%)$ & $11(23,9 \%)$ & 0 & \\
\hline & 3 & $32(36,0 \%)$ & $17(37,0 \%)$ & $2(14,3 \%)$ & \\
\hline & 4 & $12(13,5 \%)$ & $13(28,3 \%)$ & $8(57,1 \%)$ & \\
\hline & 5 & $8(9,0 \%)$ & $2(4,3 \%)$ & $2(14,3 \%)$ & \\
\hline \multirow{5}{*}{$\begin{array}{l}\text { Anatomía } \\
\text { de Grey }\end{array}$} & 1 & 7 (10,9\%) & 0 & $1(11,1 \%)$ & \multirow{5}{*}{0,30} \\
\hline & 2 & $17(26,6 \%)$ & $4(12,9 \%)$ & $3(33,3 \%)$ & \\
\hline & 3 & $21(32,8 \%)$ & $11(35,5 \%)$ & $3(33,3 \%)$ & \\
\hline & 4 & $15(23,4 \%)$ & $12(38,7 \%)$ & $2(22,2 \%)$ & \\
\hline & 5 & $4(6,3 \%)$ & $4(12,9 \%)$ & 0 & \\
\hline
\end{tabular}

Tabla IV. Justificación de la visión de las series en función del curso.

\begin{tabular}{|c|c|c|c|c|c|}
\hline & & $\begin{array}{l}1^{\text {er. curso }} \\
(n=128)\end{array}$ & $\begin{array}{c}2 . \circ \text { curso } \\
(n=63)\end{array}$ & $\begin{array}{c}3^{\text {er. }} \text { curso } \\
(n=22)\end{array}$ & $p$ \\
\hline & Contenido médico & $1(11,1 \%)$ & $2(25,0 \%)$ & 0 & \\
\hline \multirow[t]{2}{*}{ Urgencias } & Drama/comedia & $1(11,1 \%)$ & $2(25,0 \%)$ & 0 & 0,40 \\
\hline & Ambos & $7(77,8 \%)$ & $4(50,0 \%)$ & $3(21,4 \%)$ & \\
\hline \multirow{3}{*}{$\begin{array}{l}\text { Hospital } \\
\text { Central }\end{array}$} & Contenido médico & $4(11,1 \%)$ & $3(14,3 \%)$ & 0 & \\
\hline & Drama/comedia & $11(30,6 \%)$ & $5(23,8 \%)$ & $2(25,0 \%)$ & 0,80 \\
\hline & Ambos & $21(58,3 \%)$ & $13(61,9 \%)$ & $6(75,0 \%)$ & \\
\hline \multirow{3}{*}{ House } & Contenido médico & $14(15,7 \%)$ & $8(18,2 \%)$ & $3(21,4 \%)$ & \\
\hline & Drama/comedia & $13(14,6 \%)$ & $7(15,9 \%)$ & 0 & 0,80 \\
\hline & Ambos & $62(69,7 \%)$ & $29(65,9 \%)$ & $11(78,6 \%)$ & \\
\hline \multirow{3}{*}{$\begin{array}{l}\text { Anatomía } \\
\text { de Grey }\end{array}$} & Contenido médico & $1(1,5 \%)$ & $2(5,9 \%)$ & 0 & \\
\hline & Drama/comedia & $25(38,5 \%)$ & $4(11,8 \%)$ & $2(25,0 \%)$ & 0,08 \\
\hline & Ambos & $39(60,0 \%)$ & $28(82,4 \%)$ & $6(75,0 \%)$ & \\
\hline
\end{tabular}

profesionales, tal y como son representadas en las series, pueden ser idealizadas con facilidad'. En el mismo sentido está redactado el punto 8 del infor- 
me de la Organización Médica Colegial [10]: 'Muchos adolescentes pueden decidir estudiar medicina tras ver seriales médicos. El crear falsas expectativas sobre esta población tan vulnerable conducirá a tener médicos frustrados que, si pueden, terminarán abandonando la profesión con el consiguiente gasto que ello origina a la sociedad y el daño humano individual que conlleva el fracaso de un proyecto de vida laboral de estas características'.

Otra cuestión a valorar sería si este tipo de series podrían emplearse en la formación de médicos, siempre desde un punto de vista crítico. Ya se ha utilizado en este sentido para la formación de epidemiólogos [17], con buenos resultados.

En conclusión, dado que nuestros alumnos de medicina ven con frecuencia las 'series de médicos' y las consideran científicamente aceptables, nuestro deber como responsables de su formación es desde el principio alertarlos sobre la posible información errónea que aparece en ellas y, por tanto, que deben verse con una visión crítica.

Estas series podrían utilizarse en la docencia de algunas disciplinas siempre y cuando se emplearan en el aula con una visión crítica, diferenciando lo que es realidad clínica de lo que es ficción.

Bibliografía

1. Tapper EB. Doctors on display: the evolution of television's doctors. Proc (Bayl Univ Med Cent) 2010; 23: 393-9.

2. Lacalle C. Doctors in TV fiction. Quaderns del CAC 2008; 30: 51-60.

3. Series de Televisión Española. URL: http://www.rtve.es/ television/series-tve.

4. Campillo M. Series médicas televisivas. Annals del Sagrat Cor 2007; 14: 113-6.
5. Padilla G. Las series de televisión sobre médicos (1990-2010). Tres enfoques: comunicación interpersonal, comunicación institucional, relaciones entre ética, moral y política [tesis doctoral]. Madrid: Universidad Complutense de Madrid; 2010.

6. Diem SJ. Lantos JD. Tulsky JA. Cardiopulmonary resuscitation on television -miracles and misinformation. $\mathrm{N}$ Engl J Med 1996; 334: 1578-82.

7. Gordon PN, Williamson S, Lawler PG. As seen on TV: observational study of cardiopulmonary resuscitation in British television medical dramas. BMJ 1998; 317: 780-3.

8. Van den Bulck J, Damiaans K. Cardiopulmonary resuscitation on Flemish television: challenges to the television effects hypothesis. Emerg Med J 2004; 21: 565-7.

9. Harris D. Willoughby H. Resuscitation on television: realistic or ridiculous? A quantitative observational analysis of the portrayal of cardiopulmonary resuscitation in medical drama. Resuscitation 2009; 80: 1275-9.

10. Organización Médica Colegial. Informe de la Comisión Central de Deontología sobre la imagen que los seriales médicos de televisión ofrecen sobre los médicos y la profesión médica en general. Madrid: OMC; 2007. URL: http://www. comsor.es/word/informe\%20sobre\%20seriales\%20medicos-2.pdf.

11. TNS Media. Seguimiento de medios y noticias, audiencia de TV, patrocinio deportivo, medios sociales. URL: http://www. sofresam.com.

12. Bongiovanni P, González SG, Morales V, Leal LM, Saucedo K. Análisis del impacto cultural e ideológico de la serie Grey's Anatomy: estudio de caso en el área metropolitana de Monterrey. Global Media Journal 2007; 4: 107-58.

13. Guerra-Gómez A. 'Sea desabrido, camine con un bastón'. Sobre tipos y estereotipos médicos en House. Área Abierta 2007. URL: http://www.ucm.es/BUCM/revistas/inf/15788393/ articulos/ARAB0707130005A.PDF.

14. Bernal M. Las series de médicos alientan la demanda para estudiar medicina. El Periódico, 24 de julio de 2007. p. 22-3.

15. Soria M, Guerra M, Giménez I, Escanero JF. La decisión de estudiar medicina: características. Educ Med 2006; 9: 91-7.

16. Ballenato G. Esa profesión me llama. Influencias en la elección de la carrera universitaria. Madrid: Consejo General de Colegios Oficiales de Psicólogos; 2005. URL: http://www. cop.es/colegiados/m-13106/images/ArtículoCarrera.pdf.

17. Ostbye T, Miller B, Keller H. Throw that epidemiologist out of the emergency room! Using the television series ER as a vehicle for teaching methodologists about medical issues. J Clin Epidemiol 1997; 50: 1183-6. 